\title{
Impact of Tumor-shrinking Decoction (TSD) and Its Disassembled Prescriptions in Blood Serum on Gene Expression in Uterine Fibroid Cells
}

\author{
Wei Meng ${ }^{1, *}$, Xiaohua $\mathrm{Li}^{2}$, Wai Ling $\operatorname{Lin}^{1}$, Lei $\operatorname{Tan}^{3}$, Li Dong ${ }^{3}$ \\ ${ }^{1}$ School of Chinese Medicine, LKS Faculty of Medicine, the University of Hong Kong, China \\ ${ }^{2}$ Dahua Hospital, China \\ ${ }^{3}$ Yueyang Hospital, Shanghai University of TCM, China
}

Copyright $(\subset 2015$ by authors, all rights reserved. Authors agree that this article remains permanently open access under the terms of the Creative Commons Attribution License 4.0 International License

\begin{abstract}
Objective: To study the differences in vitro gene expression of uterine fibroid cells under the effect of Tumor-shrinking Decoction (TSD) and its disassembled prescriptions in blood serum, so as to investigate the target genes of TSD in uterine fibroids. Methods: TSD and its disassembled prescriptions containing blood serums were prepared. BiostarH140s microarray was used to compare the differences in gene expression of uterine fibroid cells before and after 5-day medication of TSD and its disassembled prescriptions. The calculation was based on the ratios of signal intensity of those samples, and the up and down-regulated genes were screened. Result: TSD and its disassembled prescriptions containing blood serum obviously changed the genes expression of uterine fibroid cells. There were 17 down-regulated genes and 20 up-regulated genes in tonifyingqi group (TSD-a), 26 down-regulated genes and 41 up-regulated genes in softening hardness and dissipate binds group (TSD-b), 40 down-regulated genes and 46 up-regulated genes in resolving blood stasis group (TSD-c), as well as 15 down-regulated genes and 44 up-regulated genes in TSD group. Conclusion: It is concluded that the treatment with TSD and its disassembled prescriptions can induce a variety of gene expression in uterine fibroid cells. Various genes such as p62, Mnk2, Os9, PSAP, EEF2, DCN, REL, ADAMTS1, DKK1, KLF6 and OP18 played an important role in the process. The differences in genes expression are mainly associated with the cellular signal transduction and transcription, the cell cycle, and the genes encoding protein kinase activity.
\end{abstract}

Keywords Uterine Fibroids, Leiomyoma Cells, Gene Expression, cDNA Microarray, Tumor-shrinking Decoction (TSD), Chinese Medicine

\section{Introduction}

Uterine fibroids, also known as uterine leiomyoma, are the most common benign tumors in females in the middle and later reproductive years, with a prevalence of as high as $30 \%-40 \%{ }^{[1]}$. There is about 1 out of 1000 lesions being or becoming malignant, typically as a leiomyosarcoma on histology ${ }^{[2]}$. Women with small uterine fibroids may be entirely asymptomatic. However, with tumor growth, many of them may experience irregular vaginal bleeding, heavy or painful periods, abdominal discomfort or bloating, painful defecation, back ache, urinary frequency or retention, miscarriage, premature labour and even infertility, largely depending upon the location, size and number of tumors $^{[3]}$.

Although hysterectomy and other surgical approaches have been recommended as a definitive management of uterine fibroids, surgical treatment is after all an invasive procedure and causes tissue injury ${ }^{[4]}$. Pharmacological therapies, such as gonadotrophin-releasing hormone $(\mathrm{GnRH})$ agonists and analogues, are effective in reducing the size of uterine fibroids and relieving fibroids-related symptoms. However, rapid recurrence and broad adverse side effects have largely limited the use of pharmacotherapy ${ }^{[5]}$.

In China, the use of Chinese medicine for treating uterine fibroids is a common clinical practice. Numerous Chinese medicine preparations have been shown to possess therapeutic potential in relieving uterine fibroids symptoms and shrinking the volume of fibroids without significant adverse effects, although the clinical efficacy needs to be further confirmed with rigorously designed protocols ${ }^{[6]}$.

In recent years, there are clinical studies ${ }^{[7-10]}$ showing Tumor-shrinking Decoction (TSD), previously named HualiuReceipe, attained better efficacy than conventional medicine on reduction of fibroid size and improving uterine 
fibroid symptoms, with its mild adverse effects.

In the preliminary studies ${ }^{[11]}$, BiostarH140s microarrays (containing 14,000 cDNA) were used to screen and analyze 10 pairs of differentially expressed genes of uterine fibroids and their adjacent muscle tissue. Among the 39 genes obtained with differential expression, those associated with uterine fibroids or tumor cell proliferation were: FBXO3, IFI27, KLF4, DCN, CYR61, TGFBRI, TGFBRII, and DUSP1; apoptosis-related were mainly: CTGF, IGSF4; DNA / RNA synthesis and transcription-related were: NACA, SBDS, RNase. There were also cell metabolism, differentiation and immune-related genes.

The effectiveness of the Chinese medicine decoction, $\mathrm{TSD}$, in treating uterine fibroids ${ }^{[12-14]}$ was used as the premise for this study. TSD and its disassembled prescriptions in blood serum was applied to investigate their effects in vitro uterine leiomyoma cells, so as to explore the differences in vitro gene expression of fibroid cells under the drug effect in different periods. The result of this study would provide the evidence for the mechanism of Chinese medicine in treating uterine fibroids.

\section{Materials and Methods}

\subsection{Materials}

\subsubsection{Source of Tissues}

10 pairs of fibroid tissues with the adjacent muscles were collected during the hysterectomy of symptomatic uterine fibroids of 10 subjects $(n=10)$ from Gynecology department of Yueyang Hospital of Integrated Traditional Chinese and Western Medicine, Shanghai University of Traditional Chinese Medicine. All subjects were premenopausal Chinese women aged 30-55 (mean age is 42.5) who had not received any hormonal therapy for at least 3 months prior to the surgery. The postoperative pathological diagnosis of each subject was uterine leiomyoma, and the endometrium was under proliferative phase.
With prior approval from the Institutional Review Board for Human Research of Yueyang Hospital of Integrative Medicine, Shanghai University of Traditional Chinese Medicine, all subjects signed the written consent before surgery. Under sterile conditions, two pieces of leiomyoma (approximately $0.5 * 0.5 * 0.5 \mathrm{~cm}$ ) and myometrium tissues were taken within 20-30 minutes of the extirpation of the uterus. The tissues were washed with saline, and then snap-frozen in liquid nitrogen before DNA and RNA isolation.

\subsubsection{Main Reagents}

UNIzol (Invitrogen), isopropanol, chloroform, 75\% ethanol (RNase-free), absolute ethanol (Sinopharm Chemical Reagent Co., Ltd.), Cy3 / cy5 (Amershampharmalia Biotech), reverse transcriptase (Superscript II), Oligo (dt) 18 (Gibco BRL), dNTP (PROMEGA), agarose (Sigma).

\subsubsection{Preparation of Tumor-shrinking Decoction (TSD) and Its Disassembled Prescriptions}

In this study, TSD was disassembled into three sub-groups according to different functions in the prescription. Thus, there were totally four experimental groups. The constituents of each group were shown in Table 1. All herbs were purchased from the Pharmacy of Yueyang Hospital of Integrative Medicine, Shanghai University of Traditional Chinese Medicine, and were identified and authenticated by the head of the department. The voucher specimens were stored in the laboratory. According to Pharmacopoeia of China (2005), the extraction of each component herb was weighed according to the classic percentage, and mixed well with the other extractions. The mixture was soaked in distilled water for $30 \mathrm{mins}$, and then boiled in 8 folds of volume of water for $1 \mathrm{hr}$. It was extracted twice. This preparation method followed the ancient method ${ }^{[15]}$. The supernatant was condensed to concentration of $3.9 \mathrm{~g} / \mathrm{mL}$ by water bath. The concentration of TSD and the sub-groups were expressed in total dry weight of the crude herbs per milliliter in the decoctions.

Table 1. Constituents of TSD

\begin{tabular}{|c|c|c|c|c|}
\hline $\begin{array}{c}\text { Voucher } \\
\text { number }\end{array}$ & Chinese name & Botanical name & $\begin{array}{c}\text { Weight } \\
(\mathrm{g})\end{array}$ & $\begin{array}{c}\text { Sub-G } \\
\text { roup }\end{array}$ \\
\hline A001 & Huangshi & Astragalus & $30 \mathrm{~g}$ & TSD-a \\
\hline A002 & Yiyiren & Coixlacrymajobi & $30 \mathrm{~g}$ & TSD-a \\
\hline A003 & Xiakucao & Prunella & $30 \mathrm{~g}$ & TSD-b \\
\hline A004 & Ostrea Concha & Oyster shell & $30 \mathrm{~g}$ & TSD-b \\
\hline A005 & Jianghuang & Turmeric & $30 \mathrm{~g}$ & TSD-c \\
\hline A006 & Liujinu & Siphonostegia & $15 \mathrm{~g}$ & TSD-c \\
\hline A007 & Shuizhi & Leech & $10 \mathrm{~g}$ & TSD-c \\
\hline A008 & Tubiechong & Eupolyphaga & $30 \mathrm{~g}$ & TSD-c \\
\hline
\end{tabular}

TSD-a: Tonifying qi group

TSD-b: Softening hardness and dissipate binds group

TSD-c: Resolving blood stasis group

TSD-d: Tumor-shrinking Decoction group (i.e. TSD-a + TSD-b + TSD-c) 


\subsection{Methods}

\subsubsection{Animal Grouping and Administration of TSD}

All animal procedures were conformed to Guidelines of Beijing Municipality on the Review of Welfare and Ethical of Laboratory Animals (2005). Fifty clean female Sprague-Dawley rats (weighing $200 \pm 20 \mathrm{~g}$, not yet copulated) were offered by Experimental Animal Center of Shanghai University of Traditional Chinese Medicine. They were randomized into five groups (10 rats each group), including four treatment groups (TSD-a/ TSD-b/ TSD-c/ TSD-d) and one control group (without any treatment). The rats had the adaptive feeding for 3 days. Then, they were under preoperative fasting for $12 \mathrm{hrs}$, only water was given. After intraperitoneal injection of ketamine $(100 \mathrm{mg} / \mathrm{kg})$ for anesthesia, the rats were underwent bilateral ovariectomy. After the operation, intramuscular injection of penicillin 200,000 u, Bid, was given for 3 consecutive days. On $7^{\text {th }}$ day after the operation, the treatment groups started to have intragastric administration of the TSD-a, TSD-b, TSD-c and TSD-d decoctions respectively. The dosage was equivalent to 6 times of the human clinical dose, two times per day. The control group, NS, was administered with normal saline (NS) with the same volume and procedure.

- Tonifyingqi (TSD-a) group: $6 \mathrm{~g} / \mathrm{kg} / \mathrm{d}$, intragastric administration, Bid

- Softening hardness and dissipate binds (TSD-b) group: $6 \mathrm{~g} / \mathrm{kg} / \mathrm{d}$, intragastric administration, Bid

- Resolving blood stasis (TSD-c) group: $6 \mathrm{~g} / \mathrm{kg} / \mathrm{d}$, intragastric administration, Bid

- Tumor-shrinking Decoction (TSD-d) group: concentrated to $18 \mathrm{~g} / \mathrm{kg} / \mathrm{d}$, intragastric administration, Bid

- Control (NS) group: $6 \mathrm{~g} / \mathrm{kg} / \mathrm{d}$, intragastric administration, Bid

For each group, the intragastric administration was given for 5 consecutive days. On $5^{\text {th }}$ day, the rats were fasting (only water was given) for $12 \mathrm{hrs}$ before the first administration of decoctions. The rats were administered two times at an interval of $1 \mathrm{hr}$.

\subsubsection{Preparation of Drug Serum}

One after the last administration, abdominal aortic blood was going to be collected. The rats were anesthetized by intraperitoneal injection of ketamine $(80-100 \mathrm{mg} / \mathrm{kg})$. Under sterile conditions, the abdominal cavity of each rat was opened to fully expose the abdominal aorta. The blood was collected and slowly poured into a $15 \mathrm{ml}$ centrifuge tube. After standing for 4-6hrs, the serum was obtained by centrifugation(3000r for $15 \mathrm{~min}$ ). The serum of each group was termed as TSD-containing serum or NS- containing serum (TSDCS-a, TSDCS-b, TSDCS-c, TSDCS-d, NSCS), inactivated by $56^{\circ} \mathrm{C}$ water bath for $30 \mathrm{~min}$, and filtered through the $0.22 \mu \mathrm{m}$ membrane for sterilization. Equivalent amount of serum $(1 \mathrm{ml})$ was filled in each cryotubes, and stored at $-70^{\circ} \mathrm{C}$ for later use.

\subsubsection{Primary Culture of Uterine Fibroid Cells}

\subsubsection{Isolation and Culture}

D-hank's solution was prepared by $5 \%$ collagenase (Type I) + digestive juice with $0.1 \%$ trypsin, it was filtered with a0.22 $\mu \mathrm{m}$ pore size sterile disposable filter when it was fully dissolved. Then, each fibroid tissue was washed in the sterile D-hank's solution for several times, and cut into pieces $\left(0.5-1.0 \mathrm{~mm}^{3}\right.$ in size $)$ in serum-free DMEM culture medium. The minced tissue was incubated in DMEM culture medium with $0.125 \%$ of collagenase (Type I), and stirred, shaked, digested, filtrated and centrifuged at $37 \square$. The supernatants were discarded after centrifugation; DMEM culture medium was added and the cell suspension was centrifuged again. After that, $15 \%$ fetal bovine serum (FBS) was added into DMEM medium, and it turned into the cell suspension by gently pipetting. The cells viability was examined by $0.4 \%$ Trypan Blue. Those with more than $95 \%$ were the living cells. After adjusting the cells density into $1 \times 10^{4} / \mathrm{ml}$, the cells were inoculated into the cell culture flasks, and cultured in $5 \% \mathrm{CO}^{2}$ incubator at $37^{\circ} \mathrm{C}$.

\subsubsection{Observation on the Growth of Cells}

The primary isolated cultured cells appeared adherent growth after 24 hours; the cells were elongated or spindle-shaped visibly under the microscope with good refraction (see Figure 1). The cell growth was passaged in 4-5 days.

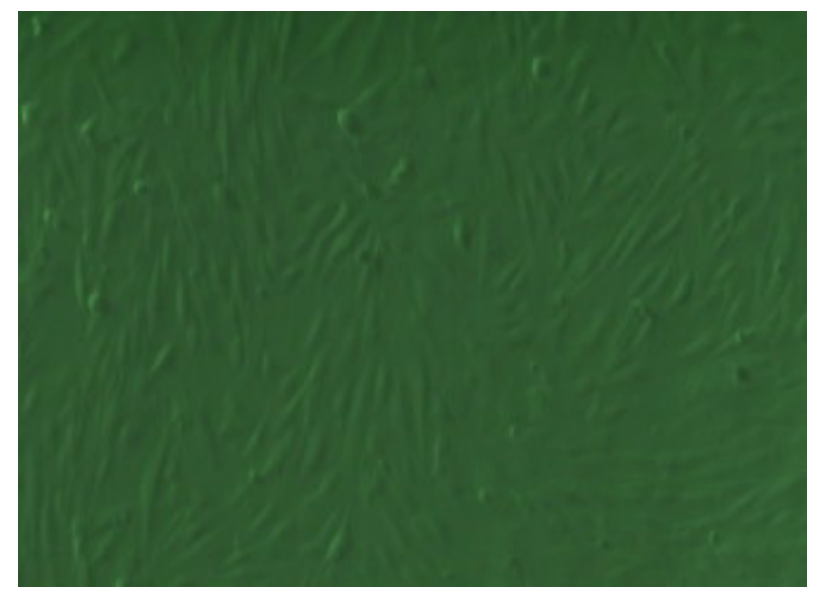

$40 \times$

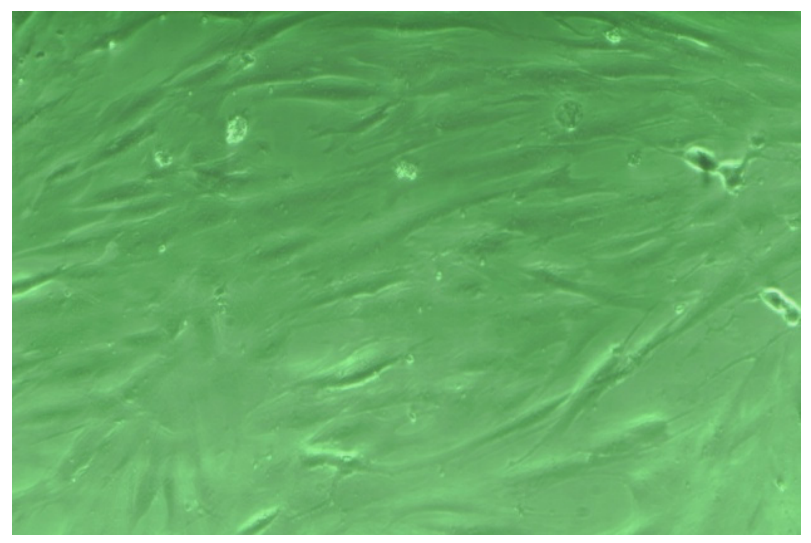

$200 \times$

Figure 1. Images of uterine fibroid cells in primary culture 


\subsubsection{Cells Passaging}

When the cells grew and reached $80 \%$ confluence, the old culture medium was discarded and $1 \mathrm{ml}$ of $0.25 \%$ trypsin was added to the culture flask. After gently shaking, the culture was discarded again. $2 \mathrm{ml}$ of $0.25 \%$ trypsin was further added and put in the incubator for $2-5 \mathrm{~min}$. When there was cytoplasmic retraction with increased cells gap seen under the inverted microscope, $2 \mathrm{ml}$ culture medium containing serum was immediately added to terminate the digestion. By repeating gently pipetting the cells on the flask wall, the cell suspension was formed and centrifuged (700rpm, 3min). The supernatant containing trypsin was discarded. 15\% DMEM medium containing fetal bovine serum was added and pipetted to form the cell suspension with $5 * 10^{5}$ cells $/ \mathrm{ml}$ seeded at the new culture flask.

\subsubsection{Identification of Uterine Fibroid Cells}
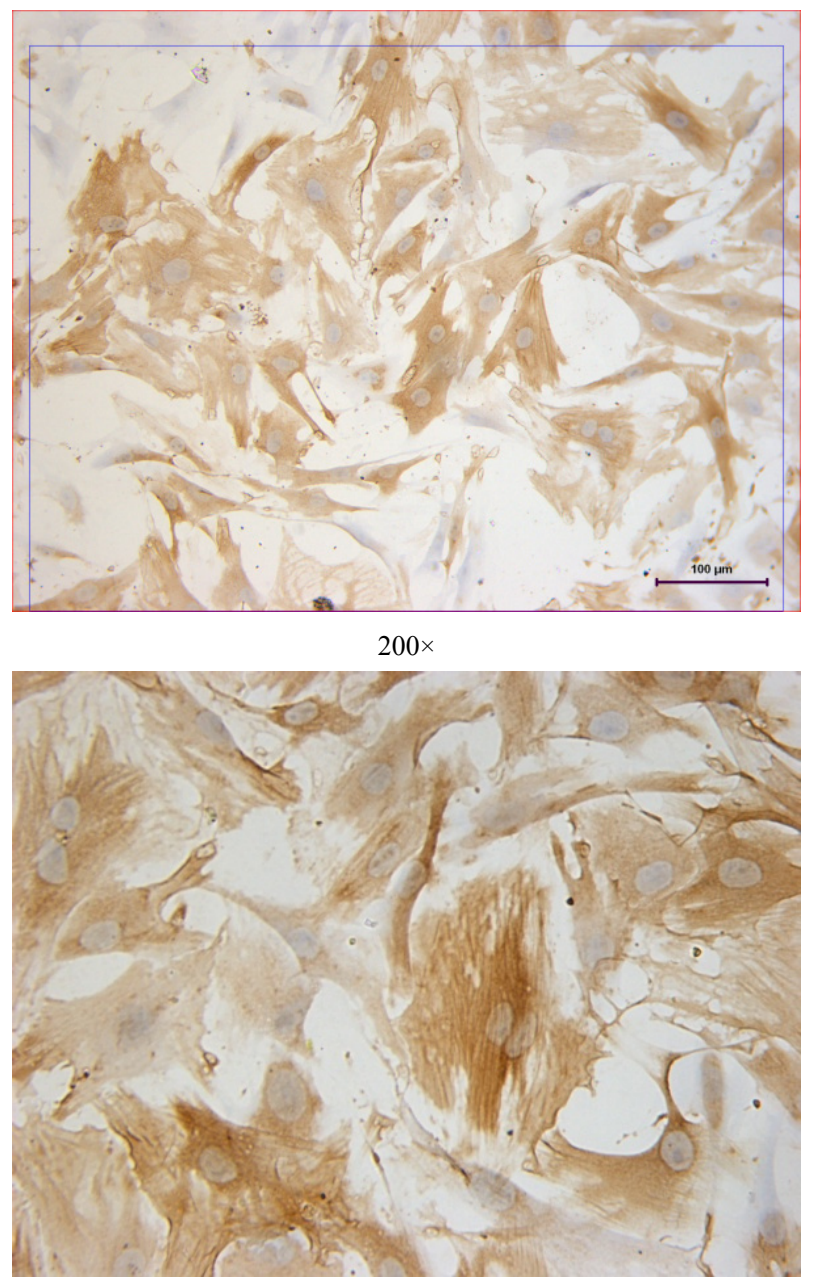

$400 \times$

NOTE: In the identification of cells under the microscope, the brown granules within the cytoplasm were in $\alpha$-actin protein expression, confirming the cultured cells were smooth muscle cells, i.e. uterine fibroid cells.

Figure 2. Imagesfor the identification of uterine fibroid cells

SP immunohistochemical method was used. After the cells being cultured and passaged, the cells morphology was stable. The anti- $\alpha$-actin staining was carried out according to the immunohistochemistry (ABC). The slide was mounted and observed under the microscope for identification. PBS method was used to replace primary antibody in the negative control. For the cells identification, see Figure 2.

\subsubsection{Collection of the Uterine Fibroid Cells}

The 3rd generation of leiomyoma cells with vigorous growth were divided into 5 groups $(\mathrm{A}, \mathrm{B}, \mathrm{C}, \mathrm{D}, \mathrm{E})$, and the five prepared serums (TSDCS-a, TSDCS-b, TSDCS-c, TSDCS-d, NSCS) were added respectively. The medium was changed once daily. After cultivation for $72 \mathrm{~h}$, trypsin digested the cells junctions. The single cell suspension was collected by centrifugation $(1000 \mathrm{r} / \mathrm{min}, 5 \mathrm{~min})$. There were about $10^{7}$ cells in each of the A, B, C, D groups while there were $4 \times 10^{7}$ cells in E group. The precipitated cells in the suspension were frozen with liquid nitrogen for the later screening of differentially expressed genes.

\subsubsection{Total RNA Extraction in the Uterine Fibroid Cells}

One step extraction method was used in extracting the total RNA in the uterine fibroid cells after the action of each drug serum. The extraction was purified and then dissolved in an appropriate amount of sterile DEPC water. After reading its absorption value in the UV spectrophotometer at $260 \mathrm{~nm}$ and $280 \mathrm{~nm}$, the concentration of RNA in each sample was calculated. The electrophoresis was carried out at the voltage of $4 \mathrm{~V} / \mathrm{cm}$. The electrophoresis should be stopped when the brophenol blue indicator run at least $2-3 \mathrm{~cm}$ of the gel length.

\subsubsection{Quality Assurance of the Extracted Total RNA}

The quality of total RNA was checked by denaturing agarose gel electrophoresis in formaldehyde. The results of gel electrophoresis are shown in Figure 3. There were three clear bands standing for 28S rRNA, 8S rRNA and 5S rRNA, which indicated that the extracted mRNA was clear and integral, with no DNA contamination nor RNA degradation, i.e. the RNA extracted was qualified.

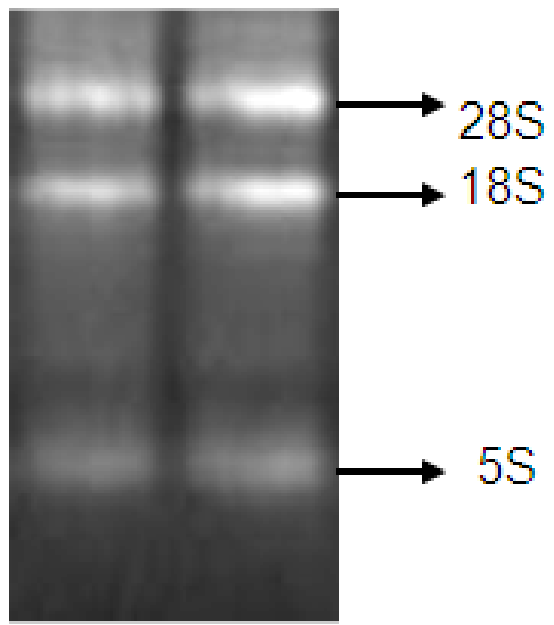

NOTE: The gel electrophoresis results indicating three clear band at the location of $5 \mathrm{~S}, 18 \mathrm{~S}$ and $28 \mathrm{~S}$.

Figure 3. Results of gel electrophoresis of total RNA in leiomyoma cells 


\subsubsection{Probe Labeling}

In the sterilized $1.5 \mathrm{~mL}$ Eppendorf tube, $23 \mathrm{ul}$ of $\mathrm{ddH}_{2}, 5 \mathrm{ul}$ of reverse primer and 30-50ug of total RNA were added in order and mixed. It was placed in the $70^{\circ} \mathrm{C}$ water bath for $10 \mathrm{~min}$. Then, it was taken out and rapidly placed in the ice. $10 \mathrm{ul}$ of reverse transcriptase buffer, 5ul of DTT and 4ul of dNTPs were added. Later, in the darkroom, $2 \mathrm{ul}$ of reverse transcriptase with $3 \mathrm{ul}$ of Cy5-dCTP or Cy3-dCTP were further added. The fibroid tissue was labeled with Cy5 while the muscle tissue was labeled with Cy3.The sample was mixed well and placed in the hand bath for $2 \mathrm{~min}$.

The Eppendorf tube was placed in the $42^{\circ} \mathrm{C}$ water bath for $2 \mathrm{hr}$. 4ul of labelling reagent I was added in the Eppendorf tube and placed in the $65^{\circ} \mathrm{C}$ water bath for $10 \mathrm{~min}$. After that, $4 \mathrm{ul}$ of labelling reagent II was added and mixed. The experimental group and control group were merged. While the light was avoided, it was under vacuum drying to about 50ul. DNA purification kit was used. The spin column was shaken vigorously in the mixer until the soluble resin suspended. The cap of the spin column top was unscrewed a quarter turn, and the sealing head of the lower end was picked off.

The spin column was placed in a $1.5 \mathrm{ml}$ Eppendorf tube, and centrifuged at 3000rpm for $1 \mathrm{~min}$. Then, the spin column was placed in another new $1.5 \mathrm{ml}$ Eppendorf tube with the top cap removed. The sample was added slowly to the middle of the resin surface with no stirring of the spin column. After centrifuging at 3000rpm for $2 \mathrm{~min}$, the purified sample was collected in the supportive Eppendorf tube. After adding $8 \mathrm{ul}$ of labeling reagent III, it was under vacuum drying.

\subsubsection{Hybridization and Washing}

$6.5 \mathrm{ul}$ of hybridization reagent I was added in the drained probe, and mixed until the probe dissolved. $6.5 \mathrm{ul}$ of hybridization reagent II was further added and mixed for later use. The pre-hybridization slide was taken out and rinsed with $\mathrm{ddH}_{2} \mathrm{O}$. The probe was denatured in the $95{ }^{\circ} \mathrm{C}$ water bath for 2 min while the slide was denatured in the $95{ }^{\circ} \mathrm{C}$ water bath for $30 \mathrm{sec}$. Then, the probe was rapidly placed on the ice, and the slide was dipped into the ethanol for $30 \mathrm{sec}$. The probe was placed on the chip with the coverslip, and sealed with Parafilm. It was put in the hybridization chamber at $42^{\circ} \mathrm{C}$ for $16-18 \mathrm{~h}$.

The slide was rinsed with $0.5 \%$ cleaning reagent I, and the coverslip was removed. Two staining jar with $0.5 \%$ cleaning reagent I $+2 \%$ cleaning reagent II $+5 \%$ cleaning reagent III were set in the $60{ }^{\circ} \mathrm{C}$ water bath. The slides were immersed in two staining jar sequentially and washed for $10 \mathrm{~min}$. Then, the slides were rinsed with $0.5 \%$ cleaning reagent $\mathrm{I}$, and dried for scanning.

\subsubsection{Scanning and Data Analysis}

(1) The chip was scanned by ScanArray 4000 Laser Scanner (General Scanning, Inc., USA) at 2 wavelengths which represented the quantity of Cy3-dUTP and Cy5-dUTP respectively. The acquired image was analyzed with
Genepix Pro 3.0 software (BioDiscovery, Inc, USA). The original signal value of each gene point on the chip was collected, including the prospective signal value and the background signal value.

(2) The actual value of the signal intensity (signal value) of the gene points with $\mathrm{Cy} 5$ or $\mathrm{Cy} 3$ was obtained by the prospect signal value subtracting the background value. All signal values which were less than 200 would be replaced by 200 in order to avoid the weak signal interference on the experimental results.

(3) To correct the systematic error in the gene points labelling system, the experimental data was under homogenization process according to two principles: 1)Both of the signal values of gene points with $\mathrm{Cy} 3$ and $\mathrm{Cy} 5$ were above 200 , or one of them was above $800 ; 2$ ) The ratio of the signal value of gene point with Cy5/the signal value of gene point with Cy3 was between 0.1 and 10 .

(4) Each ratio of $\mathrm{Cy} 3$ to $\mathrm{Cy} 5$ was computed, and the corresponding natural logarithm (r) was calculated.[r $=\ln$ (Cy5 / Cy3)]. The average value (R) of all the effective gene points was obtained, thus, the experiment homogeneous coefficient was equal to the reciprocal of R [EXP (R)].

(5) The signal values of all gene points with $\mathrm{Cy} 3$ were multiplied by the homogeneous coefficient, and the adjusted Cy3* was derived. To avoid the weak signal interference on the experimental results, all the values which were less than 200 were replaced by 200 .

(6) The ratio of the differences between the expression of each gene point was calculated $(\mathrm{Ratio}=\mathrm{Cy} 5 / \mathrm{Cy} 3 *)$.

(7) The ratio which was greater than 2 or less than 0.5 was screened out. It represented that there was greater difference between the genes and the two probes during hybridizing.

(8) The mRNA of leiomyoma cells with the interaction of TSD and its disassembled prescriptions for $72 \mathrm{~h}$ were labeled with $\mathrm{Cy} 5$ while the control one labeled with $\mathrm{Cy} 3$. In order to increase the credibility of the results, each chip was repeated the above process once, and the genes with consistent expression in both of the chips were chosen.

\section{Results}

\subsection{The Scatter Plots of the Differential Genes Expression of Leiomyoma Cells in Medicated Serum}

In the hybridization signal scan of the leiomyoma cells in the experimental group /control group, the fluorescent signal of cy5 was in green while that of cy3 was red. For one certain point with superimposed fluorescent signals, when the signal of cy3 was stronger, the point appeared as green more (down-regulating trend); when the signal of cy5 was stronger, the point appeared as red more (up-regulating trend); if the intensity of the two signals were similar, it displayed as yellow.

In Figure 4, the red signal points represented those gene points with no difference in genes expression, while the yellow signal points represented the differentially expressed genes. Moreover, the yellow signal points, which were 
beyond the red signal points and close to cy5, represented the up-regulated genes expression in leiomyoma cells in the experimental group; the yellow signal points, which were below the red signal points and close to cy 3 , represented the down-regulated genes expression in leiomyoma cells in the experimental group. From the scatter plot (see Figure 5), the differences in gene expression of leiomyoma cells in the experimental group and in the control group were shown.

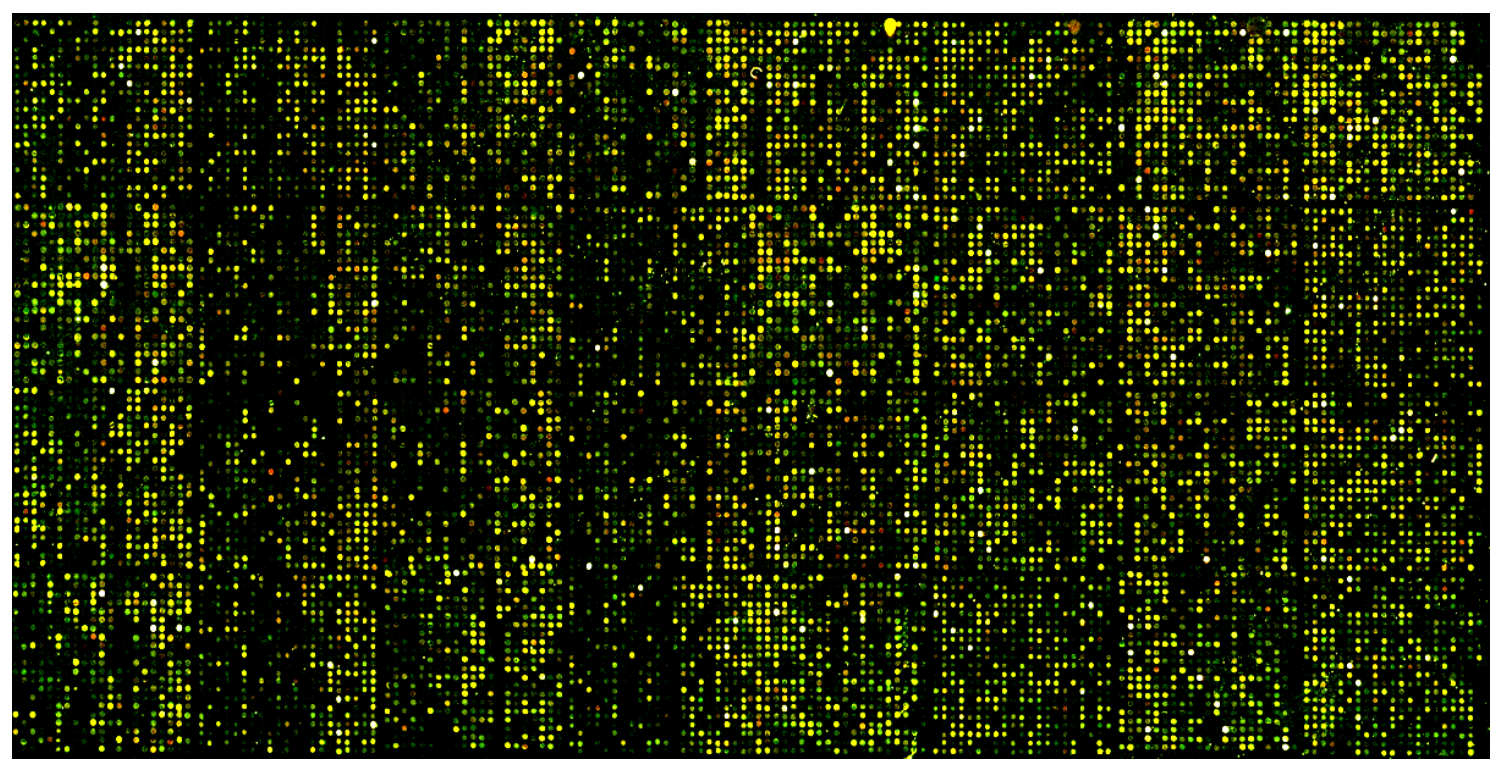

NOTE: The signal points in green represented the down-regulated genes expression in leiomyoma cells in the experimental group; The signal points in red represented the up-regulated genes expression in leiomyoma cells in the experimental group; The yellow signal points meant there were no differences in genes expression between the experimental group and the control group.

Figure 4. The hybridization signals scan of the leiomyoma cells in the experimental group /control group

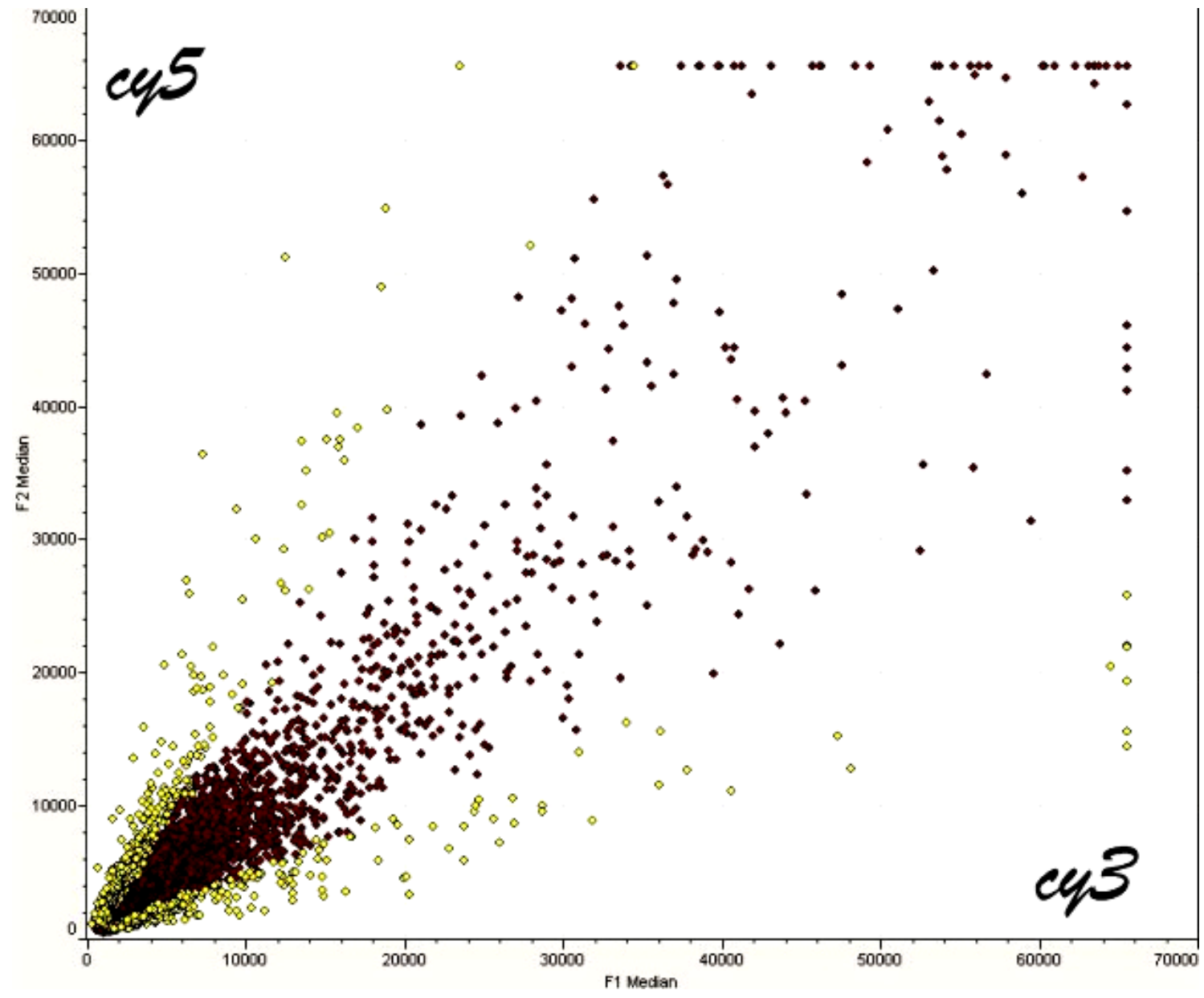




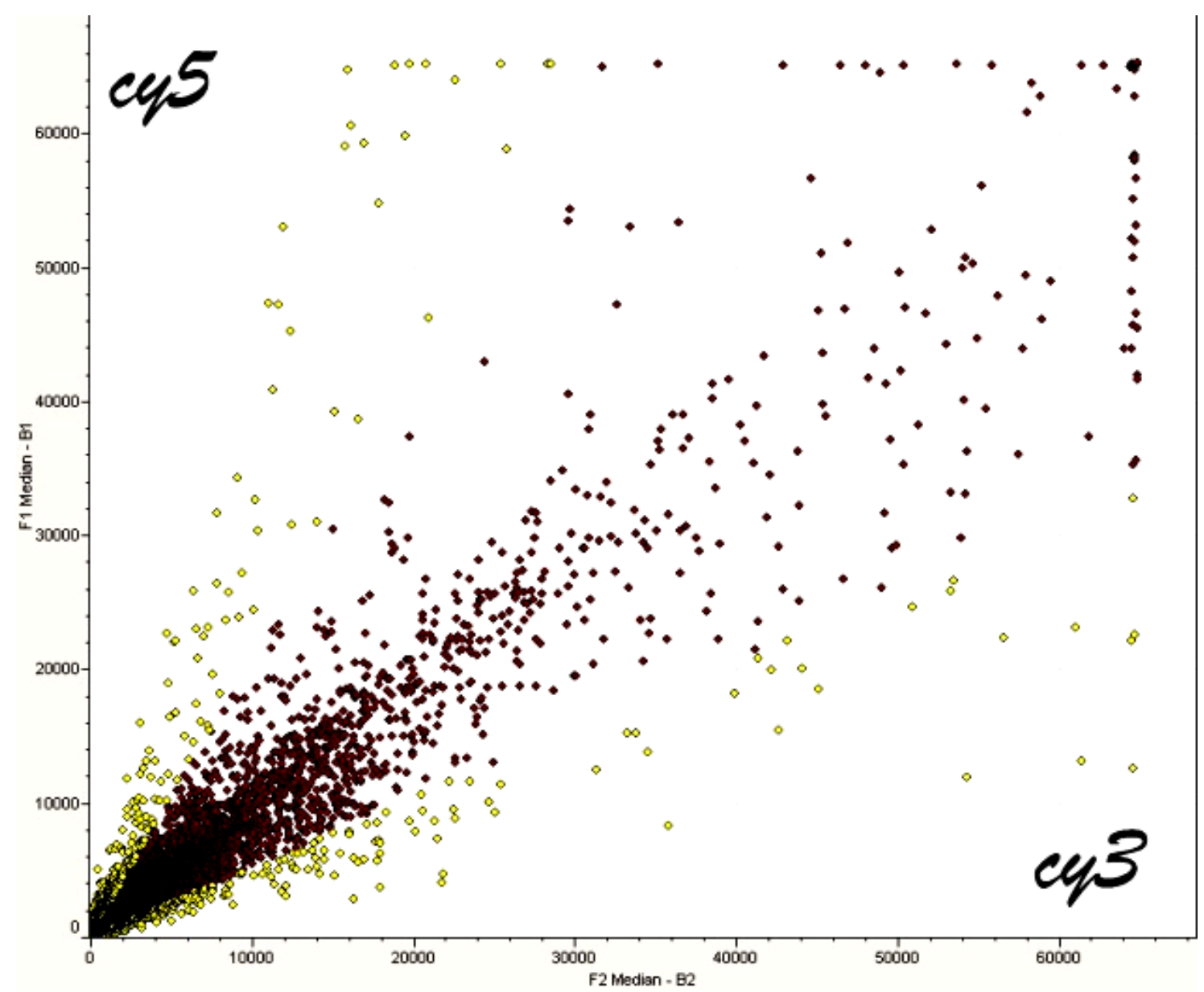

NOTE: $\mathrm{X}$-axis and $\mathrm{Y}$-axis represent the fluorescence intensity value of cy5 and cy3 respectively. Each point represents the hybridization signal of the gene point on the chip. The ratio of the red signal points was between 0.5 to 2.0 , representing the non-differential genes expression; The ratio of the yellow signal points was out of the range of 0.5 to 2.0 , representing the differential genes expression.

Figure 5. The scatter plots of hybridization signals in genes expression of leiomyoma cells in the experimental/control group

\subsection{The Change of Genes Expression in the Leiomyoma Cells after Drug Action}

The BiostarH140s microarray (with 14,000 cDNA) was applied to screen the differentially expressed genes of the uterine fibroid cells in the experimental groups (on the action of Tumor-shrinking Decoction (TSD) and its disassembled prescriptions for $72 \mathrm{~h}$ ) and control group. It was found that each experimental group had showed the obvious changes in differential genes expression in the leiomyoma cells.

For the tonifyingqi group (TSD-a), there were 17 genes showing down-regulated expression for 3 times or more (ab014460, u03106, $1202 \mathrm{~h} 06$ and m62829 were not been studied before). There were also 20 genes showing up-regulated expression for 3 times or more (3776e11 was not yet been studied). The genes with down-regulated expression were mainly related to the cell signaling, transport proteins and transcription, while the genes with up-regulated expression were mainly responsible for cell signal transduction, transcription and cell cycle.

For the softening hardness and dissipate binds group (TSD-b), there were 26 genes showing down-regulated expression for 3 times or more (u03106, 6001c08, u26727 and 129277 were not yet been studied). There were also 41 genes showing up-regulated expression for 3 times or more (4006f03, 2345h09, 2116f12, 0558e 09 and 3775e 02 were not yet been studied). The genes with down-regulated expression were mainly related to cell signaling, and some encoding involved the protein kinase activity. The genes with up-regulated expression were mainly related to cell signal transduction, transcription and cell cycle.

For the resolving blood stasis group (TSD-c), there were 40 genes showing down-regulated expression for 3 times or more (u03106,4959a04, ab014460, u23765,1202h06 and u26727 were not yet been studied). There were also 46 genes showing up-regulated expression for 3 times or more (2116f12, 0608a07, 1275b12, 0558e 09, 3775e02 and $3117 \mathrm{~d} 11$ were not yet been studied). The genes with down-regulated expression were mainly related to the protein kinase activity and transport proteins. The genes with up-regulated expression were mainly related to cell signal transduction, transcription and cell cycle.

For the tumor-shrinking Decoction group (TSD-d), there were 15 genes showing down-regulated expression for 3times or more (4959a04, 1202h06, u03106, 0309g02 and $6001 \mathrm{~d} 03$ were not yet been studied). There were also 44 genes showing up-regulated expression for 3 times or more (3776e11, 0746e 03, x05027, 0558e 09, 2116f12, 2345h09, 
$0558 \mathrm{e} 09,4006 \mathrm{f} 03,3117 \mathrm{~d} 11$ and $3775 \mathrm{e} 02$ were not yet been studied). The genes with down-regulated expression were mainly related to the protein kinase activity and signal transduction, while the genes with up-regulated expression were mainly related to cell signal transduction, transcription and cell cycle. (For details, see Table 2\&Table 3.)

Table 2. The down-regulated genes expression ( $\geq 3$ times) in group TSD-a, group TSD-b and group TSD-c compared with group TSD-d

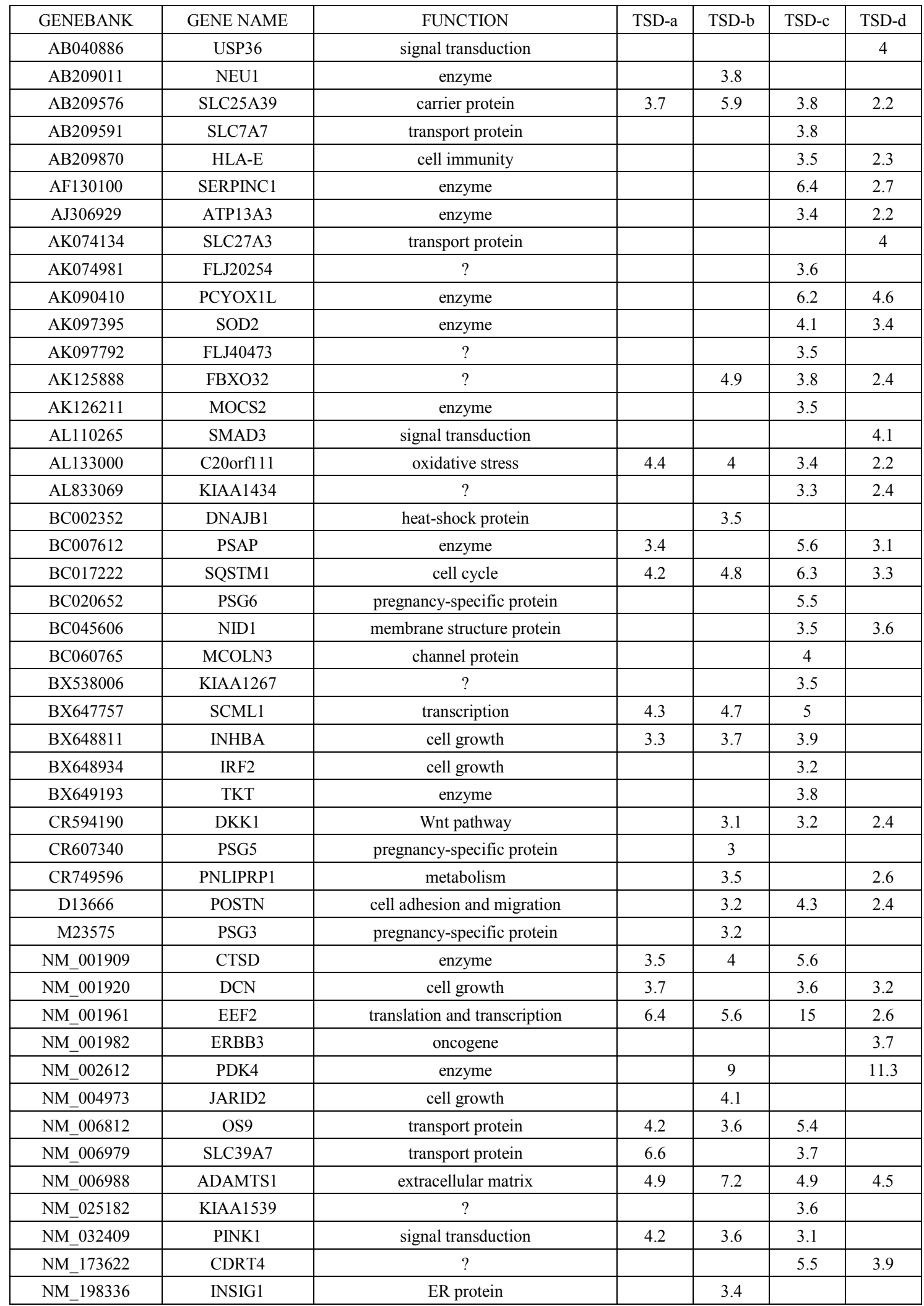


Table 3. The up-regulated genes expression ( $\geq 3$ times) in group TSD-a, group TSD-b and group TSD-c compared with group TSD-d

\begin{tabular}{|c|c|c|c|c|c|c|}
\hline GENEBANK & GENE NAME & FOUNTION & TSD-a & TSD-b & TSD-c & TSD-d \\
\hline $\mathrm{AB} 001872$ & MAP3K13 & signal transduction & & 3.3 & 3.7 & 4.2 \\
\hline $\mathrm{AB} 014572$ & KIAA0672 & $?$ & & & & 3.8 \\
\hline AB020641 & PFTK1 & signal transduction & 5.7 & & & \\
\hline $\mathrm{AB} 037715$ & KIAA1294 & $?$ & 7.5 & & & \\
\hline AB037808 & SMEK2 & signal transduction & & 3 & 3.6 & 3.5 \\
\hline AB208791 & LIG1 & enzyme & & & & 4.6 \\
\hline AB209297 & TMPO & immunity & & & 5.1 & 7.5 \\
\hline AF053306 & BUB1B & cell cycle & & & 4.1 & \\
\hline AF068846 & HNRPU & transcription & & 3.6 & 3.3 & 3.2 \\
\hline AF136175 & DDX19 & $?$ & & 3.1 & & \\
\hline AF251038 & C5orf5 & signal transduction? & & & 3.7 & \\
\hline AF288394 & C1orf19 & enzyme & 7.9 & & & \\
\hline AF332010 & IFT81 & metabolism & & 3.9 & & \\
\hline AK022972 & C6orf211 & $?$ & & & & 3.2 \\
\hline AK023169 & SRR & enzyme & & & 3.6 & \\
\hline AK024833 & KPNA2 & cell cycle & & & & 3.4 \\
\hline AK026927 & FASTKD3 & signal transduction? & 9.4 & & & 6.4 \\
\hline AK027773 & ATG4C & enzyme & & & & 3.4 \\
\hline AK055657 & HCG18 & immunity & & & 4.5 & 3.7 \\
\hline AK056803 & $\mathrm{H} 2 \mathrm{AFZ}$ & histone gene & & & & 3.3 \\
\hline AK075062 & ZNF114 & transcription & & 3.6 & 4.7 & 4.2 \\
\hline AK092622 & HNRPLL & transcription & 5.6 & & & \\
\hline AK096661 & DKFZP761M1511 & $?$ & & 4 & 3.9 & 3.5 \\
\hline AK131424 & RKHD3 & translation & & 5.4 & 3.6 & 3.8 \\
\hline AK222819 & NUSAP1 & signal transduction? & 5.1 & 5 & 4.9 & 4.9 \\
\hline AY367065 & ASPM & cell cycle & & 3.6 & & \\
\hline $\mathrm{BC} 004216$ & CBARA1 & immunity & & 4.7 & & 8.2 \\
\hline BC006135 & WIBG & $?$ & & 4.4 & 5.1 & 3.8 \\
\hline BC015621 & C14orf32 & enzyme & & & 3.9 & 3.6 \\
\hline $\mathrm{BC} 051741$ & ZNRD1 & immunity & & & 4.7 & 5.2 \\
\hline ВC052951 & LMNB1 & cell cycle & & & & 8.6 \\
\hline $\mathrm{BC} 053677$ & C5orf24 & enzyme & & & 3.8 & 2.8 \\
\hline ВC063463 & COQ3 & enzyme & & & 3.4 & \\
\hline ВC064404 & NOL11 & Transcription? & & 3.2 & & 2.9 \\
\hline ВC065295 & $\mathrm{CCDC} 25$ & $?$ & & & & 3.7 \\
\hline $\mathrm{BC} 068475$ & PNRC2 & transcription & 5.7 & & & 3.1 \\
\hline BC082272 & KCNMB3 & ion channel & & 6 & & 4.8 \\
\hline BX640701 & ZWILCH & cell cycle & & 5.5 & & 5 \\
\hline BX647885 & STMN1 & signal transduction & 5.2 & 3.7 & 4.4 & 4.3 \\
\hline BX648041 & NEDD9 & extracellular signal & & & 3.9 & \\
\hline BX648236 & PHF21A & transcription & 4.1 & & & \\
\hline BX648769 & EWSR1 & transcription & 5.8 & & & \\
\hline CR602848 & C15orf23 & $?$ & 4.2 & & & 3.3 \\
\hline CR604810 & CCNA2 & cell cycle & & & 3.5 & \\
\hline CR614015 & $\mathrm{CD} 14$ & immunity & & & & 3.9 \\
\hline CR625172 & TUBB2B & enzyme? & & & 3.1 & 3.1 \\
\hline
\end{tabular}




\begin{tabular}{|c|c|c|c|c|c|c|}
\hline CR933728 & $\mathrm{CDC} 2$ & cell cycle & 5.8 & & & 3.2 \\
\hline NM_001001323 & ATP2B1 & enzyme & & & 3.7 & \\
\hline NM_001005414 & ZWINT & cell cycle & & 8.2 & 5.2 & 5.4 \\
\hline NM_001618 & PARP1 & enzyme & & 6.1 & & 3.7 \\
\hline NM_002356 & MARCKS & signal transduction & & & & 4.2 \\
\hline NM_002388 & MCM3 & DNA replication & & & & 3.2 \\
\hline NM_002417 & MKI67 & cell cycle & & & & 6.4 \\
\hline NM_002852 & PTX3 & transcription & 23.4 & 7.6 & 5.2 & \\
\hline NM_003068 & SNAI2 & transcription & & & 3.3 & 3.3 \\
\hline NM_003151 & STAT4 & signal transduction & & 3.6 & 7.5 & 4.4 \\
\hline NM_003840 & TNFRSF10D & immunity & 4.4 & & & \\
\hline NM_003981 & PRC1 & cell cycle & & 4.1 & & 3.2 \\
\hline NM_005402 & RALA & oncogene & & & & 4.4 \\
\hline NM_005496 & SMC4 & structural protein & 5.3 & 5 & & 2.4 \\
\hline NM_005687 & FARSB & enzyme & & & 3.7 & 2.4 \\
\hline NM_006042 & HS3ST3A1 & enzyme & & & & 3.6 \\
\hline NM_006101 & NDC80 & cell cycle & & & 4.9 & 8 \\
\hline NM_006306 & SMC1A & structural protein & & 4.7 & 4.2 & 4.4 \\
\hline NM_006595 & API5 & apoptosis & & & 4.6 & 2.9 \\
\hline NM_006845 & $\mathrm{KIF} 2 \mathrm{C}$ & cell cycle & 5.7 & & & 2.9 \\
\hline NM_007111 & TFDP1 & transcription & & 3.5 & 4.4 & 3.4 \\
\hline NM_012093 & AK5 & signal transduction & & 3.3 & & \\
\hline NM_012112 & TPX2 & signal transduction & & 3.4 & & \\
\hline NM_014830 & ZBTB39 & transcription & & 3.7 & & \\
\hline NM_015426 & WDR51A & $?$ & & & 4.7 & \\
\hline NM_017785 & CCDC99 & $?$ & & 5.6 & 4.2 & 5.5 \\
\hline NM_018492 & PBK & signal transduction & & 5.4 & 3.8 & 6.2 \\
\hline NM_018947 & CYCS & apoptosis & & & & 3.5 \\
\hline NM_020374 & C12orf4 & $?$ & & 4 & 3.4 & 4.1 \\
\hline NM_021648 & TSPYL4 & $?$ & & & & 6.5 \\
\hline NM_022346 & NCAPG & structural protein & & 7.8 & 4 & 3.4 \\
\hline NM_025083 & EDC3 & transcription & & 3.8 & & \\
\hline NM_031966 & CCNB1 & cell cycle & 6.2 & 5.8 & 5 & 5.9 \\
\hline NM_032873 & STS-1 & signal transduction & & & 6.2 & \\
\hline NM_138555 & KIF23 & cell cycle & 9.8 & & & 3.3 \\
\hline NM_145323 & OSBPL3 & signal transduction & & 4.3 & 3.8 & 3.3 \\
\hline NM_145697 & NUF2 & cell cycle & & & & 3.6 \\
\hline NM_152991 & EED & transcription & & & & 3.4 \\
\hline NM_198433 & AURKA & signal transduction & & 5 & 6.2 & 5.1 \\
\hline U51869 & KLF6 & cell growth & & 4.3 & & 3 \\
\hline
\end{tabular}

NOTE: In Table 2 and Table 3, TSD-a, TSD-b, TSD-c and TSD-d groups shows the genes with three times or more differential expression. In addition, TSD-d group also lists out the genes compared with the other three groups. The corresponding values in TSD-a, TSD-b, TSD-c and TSD-d columns are the multiples of down-regulated or up-regulated differential genes expression under drug serum effect.

Through the cluster analysis of the eight chips, the genes with differential expression in the four groups were selected (i.e. these genes were consistently in up/down-regulated expression in the drug serum groups, thus, the target genes with joint action in Tumor-shrinking Decoction (TSD) and its disassembled prescriptions could be screened out). It was found that 19 genes with down-regulated expression were related to cell signaling and immune reaction; and 34 genes with up-regulated expression were involved in cell signaling, transcription and cell cycle. See Table 4 and Table 5. 
Table 4. The genes with 2 times more down-regulated expression in the four groups

\begin{tabular}{|llc|}
\hline GENEBANK & GENE NAME & FUNCTION \\
\hline AB040886 & USP36(Ubiquitin specific peptidase 36) & signal transduction \\
AB209074 & MKNK2(MAP kinase interacting serine/threonine kinase2) & signal transduction \\
AB209870 & HLA-E(major histocompatibility complex,class I,E) & cell immunity \\
AL133000 & C20orf111(chromosome 20 open reading frame 111) & transport pross responsive \\
BC007612 & PSAP(prosaposin) & signal transduction \\
BC017222 & SQSTM1(sequestosome 1) & cell membrane component \\
BC042692 & SLC44A2(solute carrier family 44, member 2) & transcription \\
BX647757 & SCML1(sex comb on midleg-like 1) & Wnt pathway \\
CR594190 & DKK1(dickkopf homolog 1) & metabolism \\
CR749596 & PNLIPRP1(pancreatic lipase-related protein 1) & cell adhesion and migration \\
D13666 & POSTN(periostin, osteoblast specific factor) & signal transduction \\
NM_000297 & PKD2(polycystic kidney disease 2) & enzyme \\
NM_001909 & CTSD(cathepsin D) & cell growth \\
NM_001920 & Decorin (DCN) & translation and transcription \\
NM_001961 & EEF2(eukaryotic translation elongation factor 2) & proto-oncogene \\
NM_002908 & REL/C-Rel(C-Rel proto-oncogene protein) & transport protein \\
NM_006812 & OS9(amplified in osteosarcoma) & transcription \\
NM_006988 & ADAMTS1(ADAM metallopeptidase with thrombospondin type1 motif,1) extracellular matrix \\
NM_017999 & RNF31(ring finger protein 31) &
\end{tabular}

Table 5. The genes with 2 times more up-regulated expression in the four groups

\begin{tabular}{|c|c|c|}
\hline GENEBANK & GENE NAME & FUNCTION \\
\hline AB001872 & MAP3K13(mitogen-activated protein kinase kinasekinase 13) & signal transduction \\
\hline AB037808 & SMEK2(SMEK homolog 2, suppressor of mek1) & signal transduction \\
\hline AB209208 & SFRS10(splicing factor, arginine/serine-rich 10) & transcription \\
\hline AB209677 & SEPT7/ CDC3 (septin 7) & cell cycle \\
\hline AF068846 & HNRPU(heterogeneous nuclear ribonucleoprotein $\mathrm{U}$ ) & transcription \\
\hline AF136175 & DDX19(DDX19-like protein variant) & $?$ \\
\hline AK026927 & FASTKD3(FAST kinase domains 3) & signal transduction? \\
\hline AK027773 & ATG4C(ATG4 autophagy related 4 homolog C) & enzyme \\
\hline AK075062 & ZNF114(zinc finger protein 114) & transcription \\
\hline AK091411 & Similar to heterogeneous nuclear ribonucleoprotein $\mathrm{H} 3$ isoform a & $?$ \\
\hline AK096661 & hypothetical protein DKFZP761M1511 & $?$ \\
\hline AK126318 & hypothetical gene supported by AK126318 & $?$ \\
\hline AK131424 & RKHD3(ring finger and $\mathrm{KH}$ domain containing 3) & translation \\
\hline AK222819 & NUSAP1(nucleolar and spindle associated protein 1) & signal transduction? \\
\hline BC006135 & WIBG(within bgen homolog) & ? \\
\hline BC035514 & TEK(TEK tyrosine kinase, endothelial) & signal transduction \\
\hline BC053677 & C5orf24(chromosome 5 open reading frame 24 ) & ? \\
\hline BX537961 & TRDMT1(tRNA aspartic acid methyltransferase 1) & enzyme \\
\hline BX641063 & DEK(DEK oncogene) & oncogene \\
\hline BX647402 & TSPAN31(tetraspanin 31 ) & signal transduction \\
\hline BX647784 & HNRPR(heterogeneous nuclear ribonucleoprotein R) & transcription \\
\hline BX647885 & STMN1(stathmin 1/oncoprotein 18) & signal transduction \\
\hline CR933728 & $\mathrm{CDC} 2$ (cell division cycle $2, \mathrm{G} 1$ to $\mathrm{S}$ and $\mathrm{G} 2$ to $\mathrm{M}$ ) & cell growth \\
\hline NM_003151 & STAT4(signal transducer and activator of transcription 4 & signal transduction \\
\hline NM_003981 & $\mathrm{PRC} 1$ (protein regulator of cytokinesis 1 ) & cell growth \\
\hline NM_005496 & SMC4(structural maintenance of chromosomes 4) & cell growth \\
\hline NM_007111 & TFDP1(transcription factor Dp-1) & transcription \\
\hline NM_012112 & TPX2(TPX2, microtubule-associated, homolog) & signal transduction \\
\hline NM_020374 & C12orf4(chromosome 12 open reading frame 4) & $?$ \\
\hline NM_031966 & CCNB1(cyclin B1) & cell growth \\
\hline NM_138555 & KIF23(kinesin family member 23) & cell growth \\
\hline $\mathrm{NM}^{-} 145323$ & OSBPL3 (oxysterol binding protein-like 3 ) & signal transduction \\
\hline U518 69 & KLF6(Kruppel-like factor 6) & cell growth \\
\hline U62027 & C3AR1(complement component 3a receptor) & immunity \\
\hline
\end{tabular}




\section{Discussion}

\subsection{The Action of Drug Serum on Target Genes}

The clinical practice has shown that the TSD was effective in treating uterine fibroids. The preliminary experimental study ${ }^{[1]}$ has suggested that the serum with Tumor-shrinking Decoction (TSD) and its disassembled prescriptions could inhibit cell proliferation of uterine fibroids. The analysis of flow cytometric apoptosis also showed that the TSD and its disassembled prescriptions could enhance the apoptosis in the fibroid cells. In order to further investigate the mechanism of the effect of TSD, we used the BiostarH140s microarray to screen the differentially expressed genes of the uterine fibroid cells after the action of serum with Tumor-shrinking Decoction (TSD) and its disassembled prescriptions for $72 \mathrm{~h}$. The categories of the screened genes included: oncogenes and tumor suppressor genes, ion channels and transport proteins, cell cycle proteins, cytoskeleton and movement, apoptosis-related proteins, DNA synthesis and repairment, recombination proteins, DNA binding and transcription factors, cell receptors, immune-relating, cell signal and transduction protein, metabolism, protein synthesis translation, development-relating, and others. We screened and compared the differentially expressed genes, and found that those genes related to cell signaling, transcription and cell cycle showed a greater change after the action of the drug serum. They may be the target genes of the pharmaceutical treatment to uterine fibroids.

As we have known, blocking the tumor cell cycle progression and promoting apoptosis play an important role in the process of tumors treatment. In this study, we found that the gene expression of SQSTM1 (sequestosome 1, also known as P62) in leiomyoma cells was significantly reduced after the effect of serum with Tumor-shrinking Decoction (TSD) and its disassembled prescriptions. P62 is highly expressed in embryos and certain tissue with high proliferation (e.g. the intestinal mucosa and skin) in adults. It is associated with the cell cycle regulation, proliferation and apoptosis ${ }^{[16]}$.P62 shows a high level of expression in a variety of tumor cells. It is associated with tumor formation and apoptosis in tumor cells. The overexpression of P62 protein may be involved in process of transformation of normal cells to malignant cells, hence, reducing its level may induce apoptosis. Also, the expression of P62 protein is associated with tumor angiogenesis ${ }^{[17]}$.Therefore, P62 is probably one of the target genes for the drug serum, and the reduction of $\mathrm{P} 62$ expression may have the association with the cycle progression and apoptosis of leiomyoma cells.

There is a close relationship between the formation of uterine fibroids and the cells differentiation. The study found that kinase 2 (MAP kinase-interacting kinase-2, Mnk2), which was interacting with the signal transduction associated genes MAPK, showed down-regulated expression after the drug serum action in the four experimental groups. Mnk2 has the property of phosphorylated eukaryotic translation initiation factor eIF4E. In addition, Mnk2 and VHL (von Hip.pel-Lindau tumor suppressor) combine with protein 1 (von Hippel-Lindau binding protein 1, VBP1), and the interactions are involved in the regulation of cell shape ${ }^{[18]}$.

In the four experimental groups, Os9 showed down-regulated expression consistently after the drug serum effect. There are studies showing Os9 expression increased in a variety of human tumor tissues; the positive rate of PSAP expression increased in primary bladder cancer, and it showed apparent down-regulated expression after the effect of the drug serum. Therefore, the reduction of Os9 and PSAP expression may impose a negative regulation to the growth of uterine fibroids. This requires further studies.

Moreover, there were some more genes downregulated expressions in all of the four groups, including: eukaryotic translation elongation factor EEF2 associated with transcription, DCN associated with the formation of fibrous connective tissue, oncogenes REL associated with cell differentiation, human histocompatibility antigen HLA-E related to cellular immunity, ADAMTS1 associated with the formation of extracellular matrix, DKK1 associated with Wnt pathway, etc. Therefore, TSD may inhibit the development of uterine fibroids through multiple pathways, such as cell proliferation, protein translation transcription, cell-mediated immunity, extracellular matrix formation and fibrosis.

The TSD serum could also down-regulate the SMAD3 gene expression. Smads pathway is the most important pathway for TGF- $\beta 1$ signal transduction. Smad2/Smad3 was the first signaling molecule for TGF- $\beta 1$ signaling, and it played an important role in the biological effects of TGF- $\beta 1$.By blocking Smad3-mediated pathway, Smad3 antisense could effectively down-regulate the TGF- $\beta$ signal transduction so as to prevent the formation of scar tissue ${ }^{[19]}$. In vivo animal experiments also showed that the removal of Smad3 gene could promote wound healing, accelerate epithelial tissue repairmen and reduce extracellular matrix deposition. In addition, the encoding of Human epidermal growth-factor receptor(HER),ERBB3 (v-erb-b2 erythroblastic leukemia viral oncogene homolog 3), showed a decreased expression on the effect of TSD drug serum. After binding with its ligand, HER was involved in cell signaling through autophosphorylation. The intracellular signals transmitted by kinase cascade, the growth and division of cells were regulated ultimately.

This study prompted that the drug serums in tonifyingqi group (TSD-a), softening hardness and dissipate binds group (TSD-b) and resolving blood stasis group(TSD-c) may also reduce the myofibroblast transformation and differentiation. INHBA and CTSD (associated with mitosis tumor and cell proliferation) played an important role in signal transduction. However, the two genes did not show significant expression in the TSD drug serum. Moreover, there were some genes showed significant differential 
expression in the TSD group (TSD-d), such as SMAD3, ERBB3, etc., but there was no significant change in the other three groups. Thus, it was confirmed that the efficacy of TSD was not simply equal to the sum of its disassembled prescriptions.

In addition, the drug serum in resolving blood stasis group(TSD-c) could obviously inhibit the expression of the interferon regulatory factor 2 (IRF2), and NIH 3 T3 cells with high expression of IRF-2 exhibited a growth characteristics with malignant proliferation and transformed cells. When these cells were injected into a nude mouse, tumors would form inside in 2-3 weeks. The drug serum of softening hardness and dissipate binds group (TSD-b) can lower the pregnancy-related specific proteins: PSG3, PSG and PSG6. These three proteins belong to the CEA gene family. However, there is lack of research on the correlation between these genes and uterine fibroids.

In the up-regulated genes, the cytoplasmic phosphoprotein OP18, associated with signal transduction, was the encoded product of oncogene STMN1 (stathmin $1 /$ oncoprotein 18). It showed reduced expression after the drug serum effect in the four groups. OP18 promotes the tubulin depolymerization. It plays an important role in the cell signal transduction related to the regulation of cell proliferation. It is the common substrate of the MAPK family and cyclin-dependent kinase $\mathrm{Cdc} 2$ family. Due to certain common channels of the signaling pathways for cell differentiation and proliferation, and the signaling pathway for apoptosis, OP18 may be involved in apoptosis signal transduction through the MAPK pathway and ca2 pathway. Therefore, the serum of tumor prescription drugs have uterine fibroids may regulate cell proliferation and apoptosis mediated by way OP18.Therefore, the TSD drug serum may regulate the cell proliferation and apoptosis in uterine fibroid cells OP18-mediated pathway.

In the comparison between the differential genes expression, the drug serums in the four groups could make the expression of leiomyoma cells KLF6 (Kruppel-like factor 6) increased. KLF6 is associated with growth, cell differentiation and growth-related cellular signal transduction, cell proliferation, apoptosis, and also angiogenesis. KLF6 can up-regulate the expression gene of p21 through the p53-dependent manner, and significantly inhibited cell proliferation. Therefore, TSD may suppress the proliferation of uterine fibroids through the up-regulation of KLF6 gene expression.

Furthermore, the encoding genes associated with cell proliferation including CCNB1, CDC2, KIF23, SMC4, $\mathrm{PRC} 1$ and SEPT7/CDC3 undergone significant up-regulated expression after the drug action of TSD and its dissembled prescriptions. The expression of these genes favored the cells to go through the various phases of the cell cycle and complete normal cell division.

The TSD drug serum could significantly up-regulate the gene expression of cytochrome c (CYCS). CYCS can activate caspase-9, which then activates Caspase-3. Through a series of cascades, it leads to apoptosis. The up-regulated expression of CYCS by TSD may be associated with the apoptosis of leiomyoma cells.

The genes encoded chromosome open reading frame, i.e. C12orf4, C14orf32, C15orf23, C1orf19, C5orf24, C5orf5, showed significantly up-regulated expression in the leiomyoma cells after the drug serum action of TSD and its dissembled prescription. However, whether these genes involved in the treatment process of uterine fibroids, further studies are necessary.

In conclusion, under the drug effects of TSD, there were a larger proportion of genes showing up-regulated expression in the uterine fibroid cells. But, the function and mediated signaling pathways of many genes have not been clearly studied yet. Moreover, there are many differentially expressed genes required further clarification for its role in the mechanism of TSD treatment on uterine fibroids. The results of this study suggest that cell signaling, cell cycle, transcription related genes, and the genes encoding protein kinase played an important role in the regulation of the mechanism of the study. They were most likely the major genes relevant to the drug actions of TSD and its dissembled prescriptions in treating uterine fibroids.

\section{REFERENCES}

[1] Munro MG. Uterine leiomyomas, current concepts: pathogenesis, impact on reproductive health, and medical, procedural, and surgical management. ObstetGynecolClin North Am. 2011;38:703-31.

[2] American Society of Reproductive Medicine Patient Booklet: Uterine Fibroids, 2003.

[3] Wallach EE, Vlahos NF. Uterine myomas: an overview of development, clinical features, and management. ObstetGynecol 2004;104(2): 393-406.

[4] Falcone T, Parker WH. Surgical management of leiomyomas for fertility or uterine preservation. Obstet Gynecol. 2013;121:856-68.

[5] Islam MS, Protic O, Giannubilo SR, Toti P, Tranquilli AL, Petraglia F, Castellucci M, Ciarmela P. Uterine leiomyoma: available medical treatments and new possible therapeutic options. J ClinEndocrinolMetab. 2013;98:921-34.

[6] Liu JP, Yang H, Xia Y, Cardini F. Herbal preparations for uterine fibroids. Cochrane Database Syst Rev. 2013;4:CD005292.

[7] Liu JP, Yang H, Xia Y, Cardini F. Herbal preparations for uterine fibroids. Cochrane Database Syst Rev. 2013;4:CD005292.

[8] Lu J, Meng W. Clinical observation 45 cases of YiqiHuayu treatment for uterine fibroids. Gansu TCM. 2008a;6:44-46.

[9] Lu J, Meng W. Clinical Observation of " Hualiu Recipe" in Treating 302 Cases of Hysteromyoma with Qi- Deficiency and Blood- Stasis Syndrome. Shanghai Traditional Chinese Medicine. 2008b;3:49-51. 
[10] Tan L, Meng W, Zhang TT. Predisposing factors of hysteromyoma and effect of hualiu recipe on it. ZhongguoZhong Xi Yi Jie He ZaZhi. 2011;31:635-638.

[11] Li XH, Meng W, Gui SQ. Research on the associated gene expression and the pathogenesis of uterine fibroids. Sheng Zhi Yu Bi Yun, 2007,27 (4): 241-246.

[12] Meng Wei, Ma Baozhang. Three drugs on cultured uterine leiomyoma cell proliferation and apoptosis, and regulatory factors. Traditional Chinese. 2003 (12); 21: 2046-2048

[13] Meng Wei. Zhao Weihong. Effects of methods of invigorating qi and dissolving stasis on the expression of proliferating and apoptosis of cultured human uterine leiomyoma cells. Chinese archives of traditional Chinese medicine. 2008(2); 238-240.

[14] Meng W, Ma BZ, Zhu NS. Research on Hualiu Recipe, Guizhifuling capsule and RU486 treatment for uterine fibroids. Zhong Yi Yao XueKan, 2002(11):35-38.
[15] Chen Q. Technology of research on pharmacology of traditional Chinese medicine. Beijing: People's Medical Publishing House. 2000: 145.

[16] LuscherB. Function and regulation of the transcription factors of the $\mathrm{My} / \mathrm{Max} / \mathrm{Mad}$ network, Gene, 2001, 277(1-2):1-14.

[17] Wang J, Dou KF, Zhao R. The association between P62 protein and bFGF expression, and gallbladder cancer tissue. Xi Bao Yu Fen ZhiMian Yi ZaZhi, 2004, 20(4): 461-464.

[18] Wang PZ, Wang X, Wang F. The interaction of Mnk2 and CBCvHL enzyme E3 ubiquitin ligase complex. ZhongGuoKeXue, 2005, 35(6): 537-544

[19] Wang DR, Liu W, Liu DL. The inhibition of cell proliferation of $3 \mathrm{~T} 3$ and gene expression of TGF- $\beta 1$ by Smad3 antisense. Shanghai Second Medical University. 